\title{
Relationship among Serum Homocysteine, Intercellular Adhesion Molecule-1, Monocyte Chemoattractant Protein-1, and Visual Impairment in Diabetic Macular Edema
}

\author{
Jinjing Fu and Jiali Zhu \\ Department of Ophthalmology, Yulin Second Hospital, Taiyuan, Shaanxi Province, China
}

\begin{abstract}
Objective: To determine the relationship among serum homocysteine (Hcy), intercellular adhesion molecule-1 (ICAM-1), monocyte chemoattractant protein-1 (MCP-1) and visual impairment in patients with diabetic macular edema (DME).

Study Design: Cross-sectional analytical study.

Place and Duration of Study: Yulin Second Hospital, China, from April 2018 to May 2021.

Methodology: A total of 132 patients with diabetic retinopathy (DR), complicated with DME, were selected as observation group; and 132 patients with simple DR were included in the control group. According to visual examination, patients in observation group were divided into visual disability and non-visual disability. Serum Hcy, ICAM-1, MCP-1, and other indicators were measured.

Results: Duration of diabetes, levels of serum Hcy, ICAM-1 and MCP-1 in observation group were higher than those in control group (all $p<0.001$ ). Levels of serum Hcy, ICAM-1 and MCP-1 in patients with mild, moderate and severe DME were significantly different (all $p<0.001$ ). Levels of serum Hcy, ICAM-1 and MCP-1 in patients with visual disabilities were higher than those in patients with non-visual disabilities (all $p<0.001$ ). Levels of serum Hcy, ICAM-1 and MCP-1 in visually disabled patients were higher than those in non-visual disability patients (all $p<0.001$ ).

Conclusion: Levels of serum Hcy, ICAM-1 and MCP-1 in patients with DR complicated with DME were higher than those without visual disability; and levels of HCy, ICAM-1 and MCP-1 in patients with visual disability were higher than those without visual disability. In patients with DR complicated with DME, increase of these serum markers may play an important role in visual disability.
\end{abstract}

Key Words: Diabetic retinopathy (DR), Diabetic macular edema (DME), Hcy, ICAM-1, MCP-1, Visual impairment.

How to cite this article: Fu J, Zhu J. Relationship among Serum Homocysteine, Intercellular Adhesion Molecule-1, Monocyte Chemoattractant Protein-1, and Visual Impairment in Diabetic Macular Edema. J Coll Physicians Surg Pak 2022; 32(01):57-60.

\section{INTRODUCTION}

Diabetic retinopathy (DR) is a common ocular neovascular disease caused by glucose metabolism disorder in patients with diabetes mellitus. ${ }^{1}$ DR can cause macular edema; and diabetic macular edema (DME) can aggravate retinal ischemia and hypoxia, leading to visual impairment, and even blindness in severe cases. ${ }^{2,3}$ The exact mechanism of DME is not clear. At present, it is considered that DME is a complex pathological process, caused by multiple factors. ${ }^{4,5}$

Correspondence to: Jiali Zhu, Department of Ophthalmology, Yulin Second Hospital, Taiyuan, Shaanxi Province, China

E-mail: zhujiali654@126.com

Received: September 27, 2021; Revised: November 02, 2021; Accepted: November 19, 2021

DOI: https://doi.org/10.29271/jcpsp.2022.01.57
It has been reported that the increase of homocysteine (Hcy) is a potential therapeutic target for $\mathrm{DR} .{ }^{6}$ Other studies have shown that DME is closely related to $\mathrm{Hcy}^{7}$ Intercellular adhesion molecule-1 (ICAM-1) is a marker of endothelial dysfunction, and its abnormal expression can cause endothelial injury. ${ }^{8}$ Injury of endothelial cell may be a major cause of ocular neovascular disease. Monocyte chemoattractant protein-1 (MCP-1) can promote the occurrence of DR through complex inflammatory pathways, and the decrease of MCP-1 level may be effective in alleviating the progress of DR. ${ }^{9}$ The higher the MCP-1 level in aqueous humor is, the greater the visual impairment and the higher likelihood of blindness will be. ${ }^{10}$ At present, the relationship among serum Hcy, ICAM-1, MCP-1 and visual impairment in patients with DME is still not clear.

The objective of this study was to investigate the relationship among serum Hcy, ICAM-1, MCP-1 and visual impairment in patients with DME. 


\section{METHODOLOGY}

This study was approved by the Research Ethics Committee of Yulin Second Hospital, China. A total of 132 patients with DR complicated with DME admitted to this hospital from April 2018 to May 2021 were selected as observation group. The inclusion criteria of the observation group were: patients with a definite history of diabetes; patients with a definite diagnosis of DR by fundus fluorescein angiography (FFA); met the diagnostic criteria of DME, namely optical coherence tomography (OCT) showed that central macular thickness (CMT) was greater than $250 \mu \mathrm{m}$ within the $1 \mathrm{~mm}$ radius of the fovea; patients with binocular affected; aged 18 to 75 years; and with informed consent.

The exclusion criteria for the observation group were patients with severe organ insufficiency; malignant tumor; nonvisualisation of fundus due to cataract and/or vitreous hemorrhage; macular edema caused by macular ischemia and vitreoretinal traction; previous macular laser photocoagulation and antiVEGF or glucocorticoid therapies in recent six months; eye surgery in recent six months; other fundus diseases such as uveitis, vein obstruction and macular membrane, examined by mydriasis; pregnant or lactating women; ocular infection; xerophthalmia and corneal complications.

A total of 132 patients with simple DR were included in the control group. The inclusion criteria of the control group were: patients with a definite history of diabetes; diagnosed as DR by fundus fluorescein angiography (FFA); patients with binocular affected; aged 18 to 75 years; and with informed consent. The exclusion criteria of the control group were: patients complicated with DME or glaucoma; those who had received other fundus surgery because of eye diseases; pregnant or lactating women; severe metabolic syndrome; severe allergic constitution; kidney disease; hyperplastic DR; malignant tumor; and if patients withdrew from the study midway, resulting in clinical data missing.

According to the international clinical classification of DME, the severity of DME in the observation group was classified as mild DME if there was a certain degree of thickening and hard exudation of the posterior polar retinal, but far from the macular centre. ModerateDME is determined as a certain degree ofthickening and hard exudation of the posterior polar retinal close to the centre of the macula, but not involved the macula. Severe DME refers to retinal thickening and hard exudation involved with the centre of macula. ${ }^{11,12}$

The uncorrected visual acuity of the observation group at admission was examined by the standard logarithmic visual acuity table, and the best corrected visual acuity was examined after optometry, and then the visual field was examined by Octopus 900 total visual field meter. According to the World Health Organization (WHO) standard of visual disability, it is defined as the binocular best corrected visual acuity $<0.02$ or visual field radius $<5$ degrees. ${ }^{13}$ According to the visual examination, the patients in the observation group were divided into visual disability and non-visual disability.
Fasting blood of elbow vein in the morning was collected within 24 hours after admission, and the serum Hcy, ICAM- 1 and MCP- 1 were measured by ELISA method, and compared between the observation group and the control group. The levels of serum Hcy, ICAM-1 and MCP-1 in patients with mild, moderate and severe DME were compared. The levels of serum Hcy, ICAM-1 and MCP-1 were compared between the patients with visual disabilities and those without visual disabilities.

Data processing was used by SPSS version 25 . The normality of variables was tested by Kolmogorov-Smirnov $(n \geq 50)$ or Shapiro-Wilk $(n<50)$. Measurement data conforming to normal distribution were expressed as mean \pm SD. Comparison of serum Hcy, ICAM-1 and MCP-1 in patients with mild $\square$ moderate and severe DME was used by one-way variance, and LSD-t test was used for comparison between the two of the above DME patients. Comparison of measurement data conforming to normal distribution in two groups was performed with independent sample t-test. Count data was expressed as n (\%), using the Chi-square test. $p<0.05$ indicates that the difference is statistically significant.

\section{RESULTS}

There was no significant difference in gender (male), age and BMI between observation group and control group $(p=0.802,0.381$ and 0.950 , respectively, Table I). The duration of diabetes, levels of serum Hcy, ICAM- 1 and MCP- 1 in observation group were higher than those in control group (all $p<0.001$, Tablel).

Table I: Comparison of demographic data and serum markers between observation group and control group.

\begin{tabular}{|c|c|c|c|}
\hline Parameter & $\begin{array}{c}\text { Control group } \\
(n=132)\end{array}$ & $\begin{array}{c}\text { Observation } \\
\text { group } \\
(n=132)\end{array}$ & p-value \\
\hline Male $[n(\%)]$ & 78(59.09) & $80(60.61)$ & 0.802 \\
\hline Age (years) & $59.45 \pm 8.80$ & $60.40 \pm 8.73$ & 0.381 \\
\hline BMI $\left(\mathrm{kg} / \mathrm{m}^{2}\right)$ & $24.06 \pm 3.56$ & $24.09 \pm 3.47$ & 0.950 \\
\hline $\begin{array}{l}\text { Duration of diabetes } \\
\text { (years) }\end{array}$ & $8.32 \pm 1.24$ & $9.08 \pm 1.29$ & $<0.001$ \\
\hline Serum Hcy (mg/L) & $12.53 \pm 1.32$ & $16.02 \pm 1.81$ & $<0.001$ \\
\hline Serum ICAM-1 ( $\mu \mathrm{g} / \mathrm{L})$ & $437.18 \pm 37.80$ & $493.00 \pm 31.87$ & $<0.001$ \\
\hline Serum MCP-1 (ng/L) & $36.71 \pm 3.89$ & $45.51 \pm 4.86$ & $<0.001$ \\
\hline
\end{tabular}

In observation group, there were 50 (37.88\%) cases, 47 (35.61\%) cases and $35(26.52 \%)$ cases of mild, moderate and severe DME, respectively. The levels of serum Hcy, ICAM-1 and MCP-1 in patients with mild, moderate and severe DME were significantly different (all $p<0.001$ ). The levels of serum Hcy, ICAM-1 and MCP-1 in patients with severe DME were higher than those in patients with mild and moderate DME (all $p<0.001$ ). The levels of serum Hcy, ICAM- 1 and MCP-1 in patients with moderate DME were higher than those in patients with mild DME $(p<0.001, p=$ 0.0001 and $p<0.001$, respectively, Table II).

Among the 132 patients in the observation group, 42 (31.82\%) patients were visually disabled and $90(68.18 \%)$ patients were of non-visual disability. Among the 42 visually disabled patients, 35 (83. 33\%) patients had severe DME and 7 (16.67\%) patients had moderateDME. 
Table II: Comparison of serum markers in mild, moderate and severe DME patients.

\begin{tabular}{|c|c|c|c|c|c|}
\hline Index & Mild DME patients $(n=50)$ & $\begin{array}{l}\text { Moderate DME patients } \\
\qquad(\mathrm{n}=47)\end{array}$ & $\begin{array}{c}\text { Severe DME } \\
\text { patients }(n=35)\end{array}$ & $\begin{array}{l}\text { Visually disabled } \\
\text { patients }(n=42)\end{array}$ & $\begin{array}{c}\text { Non-visual } \\
\text { disability } \\
\text { patients } \\
(n=90)\end{array}$ \\
\hline Serum Hcy (mg/L) & $14.30 \pm 0.95$ & $16.15 \pm 0.72$ & $18.29 \pm 0.92$ & $18.00 \pm 108$ & $15.09 \pm 1.23$ \\
\hline Serum ICAM-1 ( $\mu \mathrm{g} / \mathrm{L})$ & $474.49 \pm 31.66$ & $492.92 \pm 21.92$ & $519.56 \pm 24.28$ & $517.27 \pm 23.53$ & $481.68 \pm 28.86$ \\
\hline Serum MCP-1 (ng/L) & $40.47 \pm 2.71$ & $47.04 \pm 2.11$ & $50.67 \pm 2.56$ & $50.26 \pm 2.57$ & $43.30 \pm 4.02$ \\
\hline
\end{tabular}

Among the 90 patients with non-visual impairment, 50 (55.56\%) patients had mild DME and $40(44.44 \%)$ patients had moderate DME. The levels of serum Hcy, ICAM-1 and MCP-1 in visually disabled patients were higher than those in non-visual disability patients (all $p<0.001$ ).

\section{DISCUSSION}

DME can cause different degrees of visual impairment in diabetic patients. It is the most common factor in visual impairment and blindness in patients with DR. ${ }^{14}$ The results of this study showed that the duration of diabetes in the observation group was higher than that in the control group. It is suggested that the incidence of DME is closely related to the duration of diabetes, and the longer the course of diabetes is, the higher the incidence will be. This conclusion is basically consistent with the results of previous studies. ${ }^{15}$

Studies report that the increase of serum Hcy level may be a risk factor for DR. ${ }^{16}$ One study has shown that Hcy can be used as a biomarker for DR screening, and targeted Hcy clearance may be a therapeutic target for DR in the future. ${ }^{17}$ Another study has found that patients with proliferative DR have higher serum Hcy levels than those without DR, but the difference is not statistically significant. ${ }^{18}$ Some studies have confirmed that blood Hcy is associated with macular edema in type 2 diabetes mellitus. ${ }^{19}$ Some studies have shown that the level of ICAM-1 in aqueous humor of patients with DME is high. ${ }^{20}$ ICAM-1 is considered as a key biomarker in DME and is associated with macular size in patients with DME. ${ }^{21,22}$ The increased level of MCP-1 in eyes plays an important role in the pathogenesis of DR. Serum MCP-1 may be an important marker for diagnosis and treatment of DME. ${ }^{23}$ The results of this study showed that the levels of serum Hcy, ICAM-1 and MCP-1 in the observation group were higher than those in the control group. It suggested that the levels of serum Hcy, ICAM-1 and MCP-1 in patients with DR and DME were higher than those in patients with simple DR. It was found that in the observation group, all patients with severe DME and some patients with moderate DME were visually disabled, while none patients with mild DME had visual disability. The results showed that the visual acuity of patients with DR complicated with DME decreased significantly with the aggravation of DME. The reason may be that the more severe the degree of DME lesion is, the greater the degree of involvement in macular fovea will be, nerve cells are more prone to degeneration, and thus the visual impairment is more serious. Previous studies have also shown that the more serious the degree of DME lesion is, the more significantly the visual acuity of the patients will decrease. ${ }^{24}$ It can be seen that it is very important to understand the severity of DME early to treat the DME timely and reduce the visual disability. When DME occurs, controlling the development of DME is the key to reduce the visual disability.

Further analysis showed that the levels of serum Hcy, ICAM-1 and MCP-1 in patients with visual disabilities were higher than those in patients with non-visual disabilities. It suggested that serum Hcy, ICAM-1 and MCP-1 may be closely related to visual disability in patients with DR complicated with DME. Previous studies have shown that down-regulation of serum MCP-1 can help improve the best corrected visual acuity of DME patients. MCP-1 inhibitor may provide a new treatment method for DME. ${ }^{25}$ It can be seen that detecting levels of serum Hcy, ICAM-1 and MCP-1 is helpful to the early detection of visual disability in patients with DR complicated with DME, and the inhibition of Hcy, ICAM-1 and MCP-1 levels may help improve visual acuity in patients with DR complicated with DME.

As the sample size is small in this study, the conclusions need to be further verified. In future prospective studies, it is necessary to further verify and reveal the relationship among serum Hcy, ICAM-1, MCP-1, and visual disability in patients with DR complicated with DME.

\section{CONCLUSION}

Levels of serum Hcy, ICAM-1, and MCP-1 in patients with DR complicated with DME were higher than those without visual disability; and levels of Hcy, ICAM-1, and MCP-1 in patients with visual disability were higher than those without visual disability. In patients with DR complicated with DME, the increase of these serum markers may play an important role in visual disability.

\section{ETHICAL APPROVAL:}

This study was approved by Research Ethics Committee of Yulin Second Hospital, China.

\section{PATIENTS' CONSENT:}

Written informed consents were obtained from all participants.

\section{CONFLICT OF INTEREST:}

The authors declared no conflict of interest.

\section{AUTHORS' CONTRIBUTION:}

JF: Conception and design, analysis or interpretation of data for the work, discussion, drafting.

JZ: Literature search, discussion and revision of the manuscript. 


\section{REFERENCES}

1. Seewoodhary M. An overview of diabetic retinopathy and other ocular complications of diabetes mellitus. Nurs Stand 2021; 36(7):71-6. doi: 10.7748/ns.2021.e11696.

2. Noma H, Yasuda K, Shimura M. Involvement of cytokines in the pathogenesis of diabetic macular edema. Int J Mol Sci 2021; 22(7):3427-43. doi: 10.3390/ijms22073427.

3. Mateos-Olivares M, García-Onrubia L, Valentín-Bravo FJ, González-Sarmiento R, Lopez-Galvez M, Pastor JC, et al. Rho-Kinase inhibitors for the treatment of refractory diabetic macular oedema. Cells 2021; 10(7):1683-705. doi: 10.3390/cells10071683.

4. Gurung RL, Fitz Gerald LM, Mc Comish BJ, Verma N, Burdon $\mathrm{KP}$. Identifying genetic risk factors for diabetic macular edema and the response to treatment. J Diabetes Res 2020; 2020:5016916. doi: 10.1155/2020/5016916.

5. Kim S, Park JS, Lee J, Lee KK, Park OS, Choi HS, et al. The DME demethylase regulates sporophyte gene expression, cell proliferation, differentiation, and meristem resurrection. PNAS 2021; 118(29):e2026806118-27. doi:10.1073/pnas. 2026806118.

6. Shi C, Wang P, Airen S. Brown C, Liu Z, Townsend JH, et al. Nutritional and medical food therapies for diabetic retinopathy. Eye Vis 2020; 7(33)33-48. doi:10.1186/s40662020-00199-y.

7. Dong N, Shi H, Tang X. Plasma homocysteine levels are associated with macular thickness in type 2 diabetes without diabetic macular edema. Int Ophthalmol 2018; 38(2):737-46. doi: 10.1007/s10792-017-0528-0.

8. Habas K, Shang L. Alterations in intercellular adhesion molecule 1 (ICAM-1) and vascular cell adhesion molecule 1 (VCAM-1) in human endothelial cells. Tissue Cell 2018; 54:139-43. doi: 10.1016/j.tice.2018.09.002.

9. Kher M, Beri S, Rehan HS, Prakash A, Gupta LK. Effect of metformin and insulin combination on monocyte chemoattractant protein-1 and cathepsin-D in type 2 diabetes mellitus. Diabetes Metab Syndr 2020; 14(6):1703-10. doi: 10.1016/j.dsx.2020.08.016.

10. Song W, Zhao S, Ren B, Zhi Y. Relationships between macular edema and aqueous humor TGF-beta 2, FGF, MCP-1 levels in type 2 diabetes mellitus patients after cataract surgery. J Clin Ophthalmol 2016; 24(5):385-7. doi:10. 3969/j.issn.1006-8422.2016.05.001.

11. Wu L, Fernandez-Loaiza P, Sauma J, Hernandez-Bogantes E, Masis M. Classification of diabetic retinopathy and diabetic macular edema. World J Diabetes 2013; 4(6):290-4. doi: 10.4239/wjd.v4.i6.290.

12. Hui YN, Wang L. Introduction to the international clinical classification of diabetic retinopathy and diabetic macular edema. Int Eye Science 2004; 4(1):56-9. doi:10.3969/j.issn.1672-5123.2004.01.013.

13. Xiang Q, Li F, Chen J, Luo J. Relationships between serum $\beta 2-G P I$ and Copeptin levels and visual disability in diabetic retinopathy patients. Shandong Med J 2021; 61(25):10-3.

14. Das R, Kerr R, Chakravarthy U. Dyslipidemia and diabetic macular edema: A systematic review and Meta - Analysis. Ophthalmology 2015; 122(9):1820-7.

15. Browning DJ, Stewart MW, Lee C. Diabetic macular edema: Evidence-based management. Indian J Ophthalmol 2018; 66(12):1736-50. doi: 10.4103/ijo.IJO_1240_18.

16. Ali M, Zeba-un-Naher, Akhanda MAH, Ali S, Haque MS. Serum homocysteine level among type-2 diabetic patients with and without diabetic retinopathy. Acta Scientific Med Sci 2019; 3(8):23-31.

17. Tawfik A, Mohamed R, Elsherbiny NM, DeAngelis MM, Bartoli M, Al-Shabrawey M. Homocysteine: A potential biomarker for diabetic retinopathy. J Clin Med 2019; 8(1):121-31. doi: 10.3390/jcm8010121.

18. Prabha G, Deepa J, Grace R, Sheeja SJ. Role of hyperhomocysteinemia in proliferative diabetic retinopathy $\square$ A case-control study. Ind J Ophthalmol 2018; 66(10): 1435-40. doi: 10.4103/ijo.IJO_350_18.

19. Li J, Zhang $H$, Shi M, Yan L, Xie M. Homocysteine is linked to macular edema in type 2 diabetes. Curr Eye Res 2014; 39(7):730-5. doi: 10.3109/02713683.2013.877933.

20. Park YG, Jee D, Kwon JW. Aqueous humor cytokine levels in diabetic macular edema patients with cotton-wool spots. J Diabetes Res 2019; 2019: 8137417. doi: 10.1155/2019/ 8137417.

21. Hillier RJ, Ojaimi E, Wong DT, Mak MY, Berger AR, Kohly RP, et al. Aqueous humor cytokine levels as biomarkers of disease severity in diabetic macular edema. Retina 2017; 37(4):761-9.doi: 10.1097/IAE.0000000000001210.

22. Kwan CC, Fawzi AA. Imaging and biomarkers in diabetic macular edema and diabetic retinopathy. Curr Diabetes Rep 2019; 19:95-104. doi.10.1007/s11892-019-1226-2.

23. Monickaraj F, Nitta CF, McGuire P, Das A. Novel serum cytokines and chemokines in diabetic macular edema. Invest Ophthalmol Vis Sci 2016; 57:3270.

24. Liu J, Sun L, Gan LP. Relationship between the classification of diabetic macular edema and vision. J Clin Ophthalmol 2011; 19(4):333-4.

25. Sun HS, Sun HW. Effect of conbercept combined with macular grid photocoagulation on serum MCP-1 and VEGF levels in patients with diabetic macular edema. Chinese J Clin Res 2020; 33(3):363-6. doi.10.13429/j.cnki.cjcr. 2020.03.018. 